\title{
Prognostic Factors on the Graft-versus-Host Disease-Free and Relapse-Free Survival after Adult Allogeneic Hematopoietic Stem Cell Transplantation
}

\author{
Yao-Chung Liu, ${ }^{1,2}$ Sheng-Hsuan Chien, ${ }^{1,2,3,4}$ Nai-Wen Fan, ${ }^{2,4,5}$ Ming-Hung Hu, ${ }^{1,2,6}$ \\ Jyh-Pyng Gau, ${ }^{1,2}$ Chia-Jen Liu, ${ }^{1,2}$ Yuan-Bin Yu, ${ }^{1,2}$ Liang-Tsai Hsiao, \\ Tzeon-Jye Chiou, ${ }^{1,2}$ Cheng-Hwai Tzeng, ${ }^{1,2}$ Po-Min Chen, ${ }^{1,2}$ and Jin-Hwang Liu ${ }^{1,2}$ \\ ${ }^{1}$ Division of Hematology, Department of Medicine, Taipei Veterans General Hospital, Taipei 11217, Taiwan \\ ${ }^{2}$ Faculty of Medicine, National Yang-Ming University, Taipei 11221, Taiwan \\ ${ }^{3}$ Division of Hematology and Oncology, Department of Medicine, Taipei Veterans General Hospital, Taitung Branch, \\ Taitung City, Taitung County 95059, Taiwan \\ ${ }^{4}$ Institute of Clinical Medicine, National Yang-Ming University, Taipei 11221, Taiwan \\ ${ }^{5}$ Department of Ophthalmology, Taipei Veterans General Hospital, Taipei 11217, Taiwan \\ ${ }^{6}$ Department of Medicine, Cardinal Tien Hospital, New Taipei City 23148, Taiwan
}

Correspondence should be addressed to Jyh-Pyng Gau; jpgau@vghtpe.gov.tw

Received 2 December 2015; Revised 12 February 2016; Accepted 25 February 2016

Academic Editor: Jenny Persson

Copyright (C) 2016 Yao-Chung Liu et al. This is an open access article distributed under the Creative Commons Attribution License, which permits unrestricted use, distribution, and reproduction in any medium, provided the original work is properly cited.

\begin{abstract}
The cure of hematologic disorders by allogeneic hematopoietic stem cell transplantation (HSCT) is often associated with major complications resulting in poor outcome, including graft-versus-host disease (GVHD), relapse, and death. A novel composite endpoint of GVHD-free/relapse-free survival (GRFS) in which events include grades 3-4 acute GVHD, chronic GVHD requiring systemic therapy, relapse, or death is censored to completely characterize the survival without mortality or ongoing morbidity. In this regard, studies attempting to identify the prognostic factors of GRFS are quite scarce. Thus, we reviewed 377 adult patients undergoing allogeneic HSCT between 2003 and 2013. The 1- and 2-year GRFS were $40.8 \%$ and 36.5\%, respectively, significantly worse than overall survival and disease-free survival (log-rank $p<0.001$ ). European Group for Blood and Marrow Transplantation (EBMT) risk score $>2(p<0.001)$ and hematologic malignancy $(p=0.033)$ were poor prognostic factors for 1-year GRFS. For 2year GRFS, EBMT risk score $>2(p<0.001)$, being male $(p=0.028)$, and hematologic malignancy $(p=0.010)$ were significant for poor outcome. The events between 1-year GRFS and 2-year GRFS predominantly increased in relapsed patients. With prognostic factors of GRFS, we could evaluate the probability of real recovery following HSCT without ongoing morbidity.
\end{abstract}

\section{Introduction}

The treatment outcome of allogeneic hematopoietic stem cell transplantation (HSCT) for hematological disorders was determined by two major factors: transplant-related morbidity or mortality (TRM) and disease relapse [1]. There are many issues of dilemma in the management of patients receiving allogeneic HSCT. Graft-versus-host disease (GVHD) is the major cause of TRM while GVHD-associated graft-versusleukemia (GVL) effect can reduce the risk of malignancy relapse [2]. It has been well demonstrated that T-cell depletion of the allograft can reduce GVHD risk and therefore lower TRM [3]; however, this benefit is traded off by the risk of graft rejection and disease relapse. Regarding the intensity of conditioning, myeloablative conditioning regimen has lower risk of disease relapse while it increases risk of nonrelapse mortality (NRM), especially during first 100 days, as compared with reduced-intensity conditioning (RIC) $[4,5]$. Conversely, although RIC with fludarabinebased regimen has been used as a substitute in allowing 
older patients and those with comorbidities to safely undergo transplantation, relapse rates and GVHD risk were higher [6, 7]. It is often a difficult task to keep balance between control of posttransplant GVHD and the risk of disease relapse. Lifethreatening posttransplant complications related to GVHD would increase NRM $[8,9]$. Efforts at reducing GVHD with higher doses of immunosuppressive agents and steroids can also lead to excess deaths from opportunity infections, such as invasive fungal or viral infection [10-12]. It is mandatory to evaluate various pretransplant, peritransplant, and posttransplant risk factors that would influence survival in each individual being considered to undergo the procedure. The European Group for Blood and Marrow Transplantation (EBMT) risk score offers a simple tool to approach immediately pretransplant risks of HSCT [13]. This scoring system has been demonstrated to correlate with NRM, relapse risk, and overall survival after allogeneic HSCT for hematological disorders [13]. However, the current evaluation of clinical outcome focusing on survival alone cannot fully reflect the long-lasting complications associated with transplantation, especially GVHD, and no single evaluation can completely characterize cure without ongoing morbidity.

To address this issue, composite endpoints for posttransplant survival proposed by the Blood and Marrow Transplant Clinical Trials Network are applied for HSCT patients [1416]. The novel composite endpoints include grades 3-4 acute GVHD (aGVHD) and chronic GVHD (cGVHD) requiring systemic treatment, relapse, or death, and the outcome was defined as GVHD-free/relapse-free survival (GRFS) [14, 15]. To the best of our knowledge, only one article described the clinical prognostic factors and characteristics of GRFS at one year among different age groups $(\geq 21$ or $<21$ years) [16]. To further understand the real recovery from HSCT without major complication, we retrospectively reviewed 377 adult patients with hematological disorders treated with allogeneic HSCT between 2003 and 2013 in our institute and evaluated overall GRFS, overall survival (OS), and disease-free survival (DFS) at 1 and 2 years. In addition, we also used EBMT risk score to predict GRFS and tried to identify other prognostic factors influencing survival at the first 12 and 24 months. With the information, we hoped to optimize treatment outcome of the patients undergoing allogeneic HSCT.

\section{Materials and Methods}

2.1. Study Patient Population. Adult patients (age $\geq 18$ years) receiving allogeneic HSCT between January 2003 and September 2013 in our institute were recruited for analysis. All patients had been regularly followed till September 2014. Pertinent clinical data including age, gender, disease diagnosis, comorbidities, type of transplant, human leukocyte antigen (HLA) matching, conditioning regimen, GVHD, and other clinical complications were collected. DFS was defined as the time from last transplantation to relapse of the underlying disease or death; OS was defined as the time from last transplantation to death. In addition, the clinical outcome was also evaluated using a new composite endpoint of GRFS defined as absence of grade 3-4 aGVHD, systemic immunosuppressive therapy requiring cGVHD, relapse, or death for any causes during one and two years after allogeneic HSCT. The prognostic factors for 1- and 2-year GRFS were also explored. In the components of the GRFS events, all data were recognized as the first posttransplant event within 1 and 2 years. For those undergoing multiple allogeneic transplants, parameters pertinent to GRFS after last transplant were collected and analyzed. The donor source was predominantly peripheral blood stem cell in our study $(n=372)$. The retrospective review of medical records was approved by the institutional ethical committee in agreement with the Helsinki Declaration of 1975, revised in 2008.

2.2. Transplant Details and GVHD Prophylaxis. HLA-typing tests of intermediate resolution for 6 or 8 alleles (HLA-A, HLA-B, HLA-DR, or HLA-C) were used to select donors for allogeneic HSCT. Donor's types included matched sibling donor, matched unrelated marrow, related haploidentical donor, or umbilical cord blood. Accordingly, patients were categorized into fully matched group or mismatched group with mismatch in one or more alleles or antigens. Myeloablative conditioning regimens include busulfan ( $4 \mathrm{mg} / \mathrm{kg} /$ day for 4 days) combined with cyclophosphamide ( $60 \mathrm{mg} / \mathrm{kg} /$ day for 2 days) or total body irradiation (TBI) of 1200 cGy combined with cyclophosphamide $(60 \mathrm{mg} / \mathrm{kg} / \mathrm{day}$ for 2 days). Fludarabine-based RIC regimens were administered to patients of old age or with comorbidities.

Standard protocol with cyclosporine (IV $3.0 \mathrm{mg} / \mathrm{kg} /$ day in 2 split doses with adjusted trough plasma level maintained at $100-250 \mu \mathrm{g} / \mathrm{L})$ and short-term low dose methotrexate $\left(15 \mathrm{mg} / \mathrm{m}^{2}\right.$ on day +1 and then $10 \mathrm{mg} / \mathrm{m}^{2}$ on days $+3,+6$, and +11 after HSCT) were adopted for GVHD prophylaxis. In addition, recipients of unrelated donor transplants also received rabbit anti-thymocyte globulin $(2 \mathrm{mg} / \mathrm{kg} /$ day for $2-3$ days). Prophylactic antiviral therapy during transplantation was not routine to administration, because the seropositive rate of cytomegalovirus (CMV) IgG in Taiwan was very high [17] and most cases were "seropositive donors transfusion to seropositive donors." Instead, a policy of preemptive therapy was adopted. We detected the CMV viremia by real time PCR after transplantation and ganciclovir therapy was initiated when CMV copy numbers increased significantly or CMV disease developed clinically. In addition, trimethoprimsulfamethoxazole was prescribed within 3 months after engraftment and in parallel with immunosuppressive therapy for GVHD. Severity of aGVHD was graded according to the system of Glucksberg and Thomas and severity of the cGVHD was determined by NIH scoring system [18, 19]. Patients with aGVHD > overall grade 2, extensive cGVHD, or GVHD-related lung disease would receive treatment with methylprednisolone (MTP) 1-2 mg/kg/day.

2.3. Transplantation Risk Evaluation. We calculated transplantation risk based on EBMT risk scoring system [13] according to the age at HSCT, disease stage prior to transplantation, time interval from diagnosis to transplant, donor types, and donor recipient sex combination. The EBMT risk score was also used as a variable factor in our analysis. 
2.4. Statistical Analysis. Kaplan-Meier product-limit method was used for evaluation of 1- and 2-year posttransplant OS, DFS, and GRFS. The log-rank test was used to compare survival curves. We also explored potential prognostic factors of GRFS including age, gender, underlying disease diagnosis, EBMT risk score, transplant type, conditioning therapy, pretransplant comorbidities and 1- or 2-year posttransplant CMV reactivation, and posttransplant lymphoproliferative disease (PTLD). The posttransplant CMV reactivation indicated that antiviral agents administration was necessary based on the increased CMV copy numbers detected by real time polymerase chain reaction or clinical symptoms. The prognostic factors were analyzed using Cox proportional hazard models. Factors with statistical significance $(p<$ 0.05 ) upon univariate analysis were included in multivariate analysis. All statistically significant levels were set at $p<$ 0.05. Results were expressed as hazard ratio (HR) and their corresponding 95-percent confidence intervals (95\% $\mathrm{CI})$. In the analysis of age as prognostic factor, age of 35 years was adopted as cutoff value based on the results of receiver operating characteristic curve. All calculations were performed using the Statistical Package of Social Sciences software (version 18.0; SPSS Inc., Chicago, IL, USA).

\section{Results}

We retrospectively reviewed totally 377 patients receiving allogeneic HSCT between January 2003 and September 2013. The median age at HSCT was 41 years (range: 18-67). Male patients were slightly predominant $(55.4 \%)$. Hematological malignancies comprised $87.3 \%(n=329)$ of diagnosis and the rest were other nonmalignant diseases $(12.7 \%, n=48$, including 45 with severe aplastic anemia and 3 with myelodysplastic syndrome without excess blasts). Donor types included 181 matched sibling donors (48.0\%) and 196 patients $(52.0 \%)$ with matched unrelated donors or incompletely matched sibling and unrelated sources. Median duration of followup after HSCT was 554 days (range: 11-4097). Myeloablative conditioning regimen was more frequently used in the cohort $(65.8 \%)$. About half of the patients experienced posttransplant CMV reactivation within one year (49.9\%). Eventually, 10 patients had CMV pneumonitis, 2 had CMV enteritis, and 2 had CMV retinitis. Few patients suffered from PTLD (3.7\% and $4.0 \%$ within one and two years). The clinical characteristics were detailed in Table 1.

3.1. Clinical Prognostic Factors for GRFS. In the univariate regression analysis for prognostic factors, age at HSCT $(p=$ 0.011; HR: 1.446), EBMT risk score ( $p<0.001$; HR: 2.197), disease type at diagnosis ( $p=0.002$; HR: 2.244), gender ( $p=0.012$; HR: 1.419), use of fludarabine-based conditioning regimen ( $p=0.012$; HR: 1.491$)$, and posttransplant CMV reactivation ( $p=0.033$; HR: 1.335$)$ significantly impacted 1-year GRFS (Table 2). In multiple regression analysis, the prognostic factors that remained significant for GRFS at 1 year were EBMT risk score $>2(p<0.001$; HR: 1.897; 95\% CI: 1.385-2.599) and hematologic malignancy at diagnosis ( $p=0.033$; HR: 1.763; 95\% CI: 1.048-2.966).
TABLE 1: Clinical characteristics of study patients $(n=377)$.

\begin{tabular}{|c|c|c|}
\hline Patient characteristics & $n$ & $\%$ \\
\hline \multicolumn{3}{|l|}{ Gender } \\
\hline Female & 168 & 44.6 \\
\hline Male & 209 & 55.4 \\
\hline \multicolumn{3}{|l|}{ Age at HSCT } \\
\hline$\leq 35$ & 139 & 36.9 \\
\hline$>35$ & 238 & 63.1 \\
\hline \multicolumn{3}{|l|}{ EBMT risk score } \\
\hline$\leq 2$ & 155 & 41.1 \\
\hline$>2$ & 222 & 58.9 \\
\hline \multicolumn{3}{|l|}{ Indication for HSCT } \\
\hline AML/MDS & 157 & 41.7 \\
\hline MPD & 19 & 5.0 \\
\hline ALL & 74 & 19.6 \\
\hline Lymphoma & 63 & 16.7 \\
\hline $\mathrm{MM}$ & 18 & 4.8 \\
\hline SAA & 45 & 11.9 \\
\hline Others & 1 & 0.3 \\
\hline \multicolumn{3}{|l|}{ Donor relation } \\
\hline Matched sibling & 181 & 48.0 \\
\hline Nonmatched sibling & 196 & 52.0 \\
\hline \multicolumn{3}{|l|}{ Conditioning regimen } \\
\hline TBI-12 Gy based & 125 & 33.2 \\
\hline Fludarabine based & 74 & 19.6 \\
\hline Myeloablative & 248 & 65.8 \\
\hline Type 2 DM history & 15 & 4.0 \\
\hline Smoking history & 24 & 6.4 \\
\hline \multicolumn{3}{|c|}{ Posttransplant CMV reactivation } \\
\hline Within 1 year & 188 & 49.9 \\
\hline Within 2 years & 190 & 50.4 \\
\hline \multicolumn{3}{|l|}{ PTLD after HSCT } \\
\hline Within 1 year & 14 & 3.7 \\
\hline Within 2 years & 15 & 4.0 \\
\hline
\end{tabular}

HSCT: hematopoietic stem cell transplantation; MDS: myelodysplastic syndromes; EBMT: European Group for Blood and Marrow Transplantation; AML: acute myeloid leukemia; MPD: myeloproliferative disorder; ALL: acute lymphoblastic leukemia; MM: multiple myeloma; SAA: severe aplastic anemia; TBI: total body irradiation; CMV: cytomegalovirus; DM: diabetes mellitus; PTLD: posttransplant lymphoproliferative disorder.

For 2-year prognostic factors of GRFS, age at HSCT $(p=$ 0.008; HR: 1.452), EBMT risk score ( $p<0.001$; HR: 2.165), disease type at diagnosis $(p<0.001$; HR: 2.495$)$, gender $(p=$ 0.005; HR: 1.456), fludarabine-based conditioning regimen ( $p=0.009$; HR: 1.494), and posttransplant CMV reactivation $(p=0.023$; HR: 1.349) still significantly impacted 2-year GRFS (Table 3). However, in multiple regression analysis, the poor prognostic factors for 2-year GRFS were EBMT risk score $>2(p<0.001$; HR: 1.835; 95\% CI: 1.354-2.486), being male ( $p=0.028$; HR: 1.348; 95\% CI: 1.032-1.761), and hematologic malignancy at diagnosis ( $p=0.010$; HR: 1.979; 95\% CI: 1.178-3.324). 
TABLE 2: Prognostic factors for 1-year GRFS after adult allogeneic HSCT.

\begin{tabular}{|c|c|c|c|c|c|c|c|c|c|}
\hline \multirow{2}{*}{ Factors } & \multirow{2}{*}{$n$} & \multirow{2}{*}{ Events } & \multirow{2}{*}{$\%$} & \multicolumn{3}{|c|}{ Univariate analysis } & \multicolumn{3}{|c|}{ Multivariate analysis } \\
\hline & & & & HR & $95 \% \mathrm{CI}$ & $p$ value & HR & $95 \% \mathrm{CI}$ & $p$ value \\
\hline \multicolumn{10}{|l|}{ Age at HSCT } \\
\hline$\leq 35$ & 139 & 69 & 49.6 & \multirow{2}{*}{1.446} & \multirow{2}{*}{$1.087-1.923$} & \multirow{2}{*}{0.011} & \multirow{2}{*}{1.068} & \multirow{2}{*}{$0.783-1.456$} & \multirow{2}{*}{0.679} \\
\hline$>35$ & 238 & 151 & 63.4 & & & & & & \\
\hline \multicolumn{10}{|l|}{ EBMT risk score } \\
\hline$\leq 2$ & 155 & 67 & 43.2 & \multirow{2}{*}{2.197} & \multirow{2}{*}{$1.647-2.913$} & \multirow{2}{*}{$<0.001$} & \multirow{2}{*}{1.897} & \multirow{2}{*}{$1.385-2.599$} & \multirow{2}{*}{$<0.001$} \\
\hline$>2$ & 222 & 153 & 68.9 & & & & & & \\
\hline \multicolumn{10}{|l|}{ Gender } \\
\hline Female & 168 & 84 & 50.0 & \multirow{2}{*}{1.419} & \multirow{2}{*}{$1.081-1.863$} & \multirow{2}{*}{0.012} & 1.310 & $0.994-1.725$ & 0.055 \\
\hline Male & 209 & 136 & 65.1 & & & & 1.510 & $0.994-1 . / 20$ & 0.050 \\
\hline Disease type & & & & & & & & & \\
\hline Nonmalignant & 48 & 16 & 33.3 & 2.244 & $1.348-3.735$ & 0.002 & 1.763 & $1.048-2.966$ & 0.033 \\
\hline Malignant & 329 & 204 & 62.0 & 2.244 & $1.348-3.135$ & 0.002 & $1 . / 03$ & $1.040-2.900$ & 0.050 \\
\hline Disease type & & & & & & & & & \\
\hline Myeloid & 176 & 111 & 63.1 & 0.835 & $0.641-1.088$ & 0.182 & & & \\
\hline Nonmyeloid & 201 & 109 & 54.2 & 0.853 & $0.041-1.088$ & 0.182 & & & \\
\hline Conditioning & & & & & & & & & \\
\hline Nonmyeloablative & 129 & 72 & 55.8 & 1.084 & $0.818-1.436$ & 0.576 & & & \\
\hline Myeloablative & 248 & 148 & 59.7 & 1.084 & $0.818-1.430$ & $0.5 / 0$ & & & \\
\hline Conditioning & & & & & & & & & \\
\hline Others & 252 & 142 & 56.3 & 1.212 & $0.920-1.598$ & 0.172 & & & \\
\hline 12 Gy TBI based & 125 & 78 & 62.4 & 1.212 & $0.920-1.598$ & $0.1 / 2$ & & & \\
\hline Conditioning & & & & & & & & & \\
\hline Others & 303 & 168 & 55.4 & 1.491 & $1.092-2.036$ & 0.012 & 1.134 & $0.818-1.574$ & 0.450 \\
\hline Fludarabine based & 74 & 52 & 70.2 & & $1.092-2.030$ & 0.012 & & & \\
\hline Donor type & & & & & & & & & \\
\hline Matched sibling & 181 & 111 & 61.3 & 1005 & $0771-1.309$ & 0971 & & & \\
\hline Others & 196 & 109 & 55.6 & & & & & & \\
\hline Type 2 DM & & & & & & & & & \\
\hline No & 362 & 210 & 58.0 & 1.339 & $0.710-2.526$ & 0.367 & & & \\
\hline Yes & 15 & 10 & 66.7 & 1.507 & $0.110-2 . J 20$ & 0.507 & & & \\
\hline Smoking history & & & & & & & & & \\
\hline No & 353 & 204 & 57.8 & 1.348 & $0.810-2.242$ & 0.250 & & & \\
\hline Yes & 24 & 16 & 66.7 & 1.340 & $0.010-2.242$ & 0.250 & & & \\
\hline Posttransplant CMV r & & & & & & & & & \\
\hline No & 189 & 101 & 53.4 & 1.335 & $1.024-1.741$ & 0.033 & 1.160 & $0.884-1.522$ & 0.285 \\
\hline Yes & 188 & 119 & 63.3 & 1.535 & $1.024-1.741$ & 0.050 & 1.100 & $0.004-1.522$ & 0.200 \\
\hline PTLD & & & & & & & & & \\
\hline No & 363 & 212 & 58.4 & 047 & & & & & \\
\hline Yes & 14 & 8 & 57.1 & 047 & $0.51 /-2.120$ & 0.899 & & & \\
\hline
\end{tabular}

CI: confidence interval; HR: hazard ratio; EBMT: European Group for Blood and Marrow Transplantation; HSCT: hematopoietic stem cell transplantation; PTLD: posttransplant lymphoproliferative disorders; DM: diabetes mellitus; CMV: cytomegalovirus; TBI: total body irradiation.

Factors with statistical significance $(p<0.05)$ upon univariate analysis were included in multivariate analysis.

In the study, the survival rate of Kaplan-Meier curve for 1- and 2-year GRFS revealed $40.8 \%$ and $36.5 \%$, respectively, as significantly compared with DFS ( 1 year: $56.6 \%$; 2 years: $50.8 \%)$ and OS (1 year: 62.4\%; 2 years: 56.8\%) (log-rank $p<0.001$; Figures 1 and 2). In the four events of 1 - and 2-year GRFS, relapsed group was increased prominently in the 2-year GRFS (30.9\% to 32.9\%; Figure 3). For the poor prognostic factors of GRFS including being male, EBMT risk score $>2$, and hematologic malignancy at diagnosis, predominantly increased proportions in relapsed and death 
TABLE 3: Prognostic factors for 2-year GRFS after adult allogeneic HSCT.

\begin{tabular}{|c|c|c|c|c|c|c|c|c|c|}
\hline \multirow{2}{*}{ Factors } & \multirow{2}{*}{$n$} & \multirow{2}{*}{ Events } & \multirow{2}{*}{$\%$} & \multicolumn{3}{|c|}{ Univariate analysis } & \multicolumn{3}{|c|}{ Multivariate analysis } \\
\hline & & & & HR & $95 \%$ CI & $p$ value & HR & $95 \%$ CI & $p$ value \\
\hline \multicolumn{10}{|l|}{ Age at HSCT } \\
\hline$\leq 35$ & 139 & 74 & 53.2 & \multirow{2}{*}{1.452} & \multirow{2}{*}{$1.102-1.913$} & \multirow{2}{*}{0.008} & \multirow{2}{*}{1.080} & \multirow{2}{*}{$0.799-1.459$} & \multirow{2}{*}{0.617} \\
\hline$>35$ & 238 & 160 & 67.2 & & & & & & \\
\hline \multicolumn{10}{|l|}{ EBMT risk score } \\
\hline$\leq 2$ & 155 & 73 & 47.1 & \multirow{2}{*}{2.165} & \multirow{2}{*}{$1.640-2.857$} & \multirow{2}{*}{$<0.001$} & \multirow{2}{*}{1.835} & \multirow{2}{*}{$1.354-2.486$} & \multirow{2}{*}{$<0.001$} \\
\hline$>2$ & 222 & 161 & 72.7 & & & & & & \\
\hline \multicolumn{10}{|l|}{ Gender } \\
\hline Female & 168 & 89 & 53.0 & \multirow{2}{*}{1.456} & \multirow{2}{*}{$1.118-1.896$} & \multirow{2}{*}{0.005} & 1348 & $1.032-1761$ & 0.028 \\
\hline Male & 209 & 145 & 69.4 & & & & 1.348 & $1.032-1 . / 01$ & 0.028 \\
\hline Disease type & & & & & & & & & \\
\hline Nonmalignant & 48 & 16 & 33.3 & 2495 & $1500-4149$ & $<0,001$ & 1979 & $1178-3,324$ & 0.010 \\
\hline Malignant & 329 & 218 & 66.3 & 2.100 & 1.000 & 0.001 & & & \\
\hline Disease type & & & & & & & & & \\
\hline Myeloid & 176 & 117 & 66.5 & 0836 & 0 647-1081 & 0172 & & & \\
\hline Nonmyeloid & 201 & 117 & 58.2 & 0.850 & $0.04 /-1.081$ & $0.1 / 2$ & & & \\
\hline Conditioning & & & & & & & & & \\
\hline Nonmyeloablative & 129 & 75 & 58.1 & 1.135 & $0.863-1.494$ & 0.365 & & & \\
\hline Myeloablative & 248 & 159 & 64.1 & & & & & & \\
\hline Conditioning & & & & & & & & & \\
\hline Others & 252 & 150 & 59.5 & 1.246 & $0953-1627$ & 0107 & & & \\
\hline 12 Gy TBI based & 125 & 84 & 67.2 & & $0.930-1.027$ & 0.107 & & & \\
\hline Conditioning & & & & & & & & & \\
\hline Others & 303 & 179 & 59.1 & 1.494 & $1.104-2.022$ & 0.009 & 1.115 & $0.811-1.532$ & 0.503 \\
\hline Fludarabine based & 74 & 55 & 74.3 & & & & & & \\
\hline Donor type & & & & & & & & & \\
\hline Matched sibling & 181 & 119 & 65.7 & 0.976 & $0.755-1.261$ & 0.851 & & & \\
\hline Others & 196 & 115 & 58.7 & & & & & & \\
\hline Type 2 DM & & & & & & & & & \\
\hline No & 362 & 224 & 61.9 & 1.260 & $0.699-2.375$ & 0.474 & & & \\
\hline Yes & 15 & 10 & 66.7 & & & & & & \\
\hline Smoking history & & & & & & & & & \\
\hline No & 353 & 216 & 61.2 & 1.415 & $0.903-2.364$ & 0.122 & & & \\
\hline Yes & 24 & 18 & 75.0 & & & & & & \\
\hline Posttransplant CMV 1 & & & & & & & & & \\
\hline No & 187 & 107 & 57.2 & 1349 & $1042-1746$ & 0.023 & 1170 & $0899-1523$ & 0.242 \\
\hline Yes & 190 & 127 & 66.8 & & & & & & \\
\hline PTLD & & & & & & & & & \\
\hline No & 362 & 228 & 62.9 & 1463 & $0.651-3.292$ & 0.357 & & & \\
\hline Yes & 15 & 6 & 40.0 & & & & & & \\
\hline
\end{tabular}

CI: confidence interval; HR: hazard ratio; EBMT: European Group for Blood and Marrow Transplantation; HSCT: hematopoietic stem cell transplantation; PTLD: posttransplant lymphoproliferative disorders; DM: diabetes mellitus; CMV: cytomegalovirus; TBI: total body irradiation.

Factors with statistical significance $(p<0.05)$ upon univariate analysis were included in multivariate analysis.

subgroups were noted within 1 and 2 years. No marked difference was found between aGVHD and cGVHD within 1 and 2 years (Figure 3 ).

\section{Discussion}

By using the concept of GRFS defined by Blood and Marrow Transplant Clinical Trials Network, we found that $40.8 \%$ and
$36.5 \%$ of our patients could survive to 1 and 2 years, respectively, without experiencing relapse or significant GVHD. Compared to data from the Center for International Blood and Marrow Transplant Research between 2006 and 2009, our 1-year probability of GRFS was better ( $40.8 \%$ versus $23 \%$ ) [15]. In comparison to a recent article reported by Holtan et al., the 1-year OS (63\%) and DFS (53\%) were similar to our cohort (Figure 1); however, our patients had better 1-year 


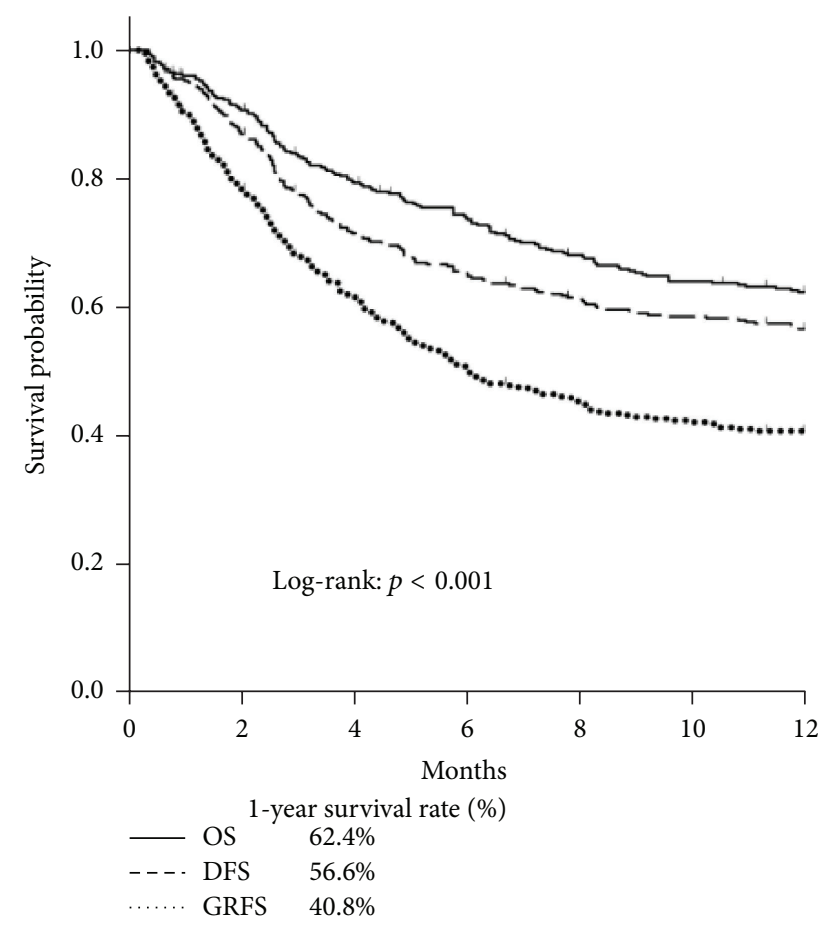

FIGURE 1: Kaplan-Meier estimates of 1-year GRFS.

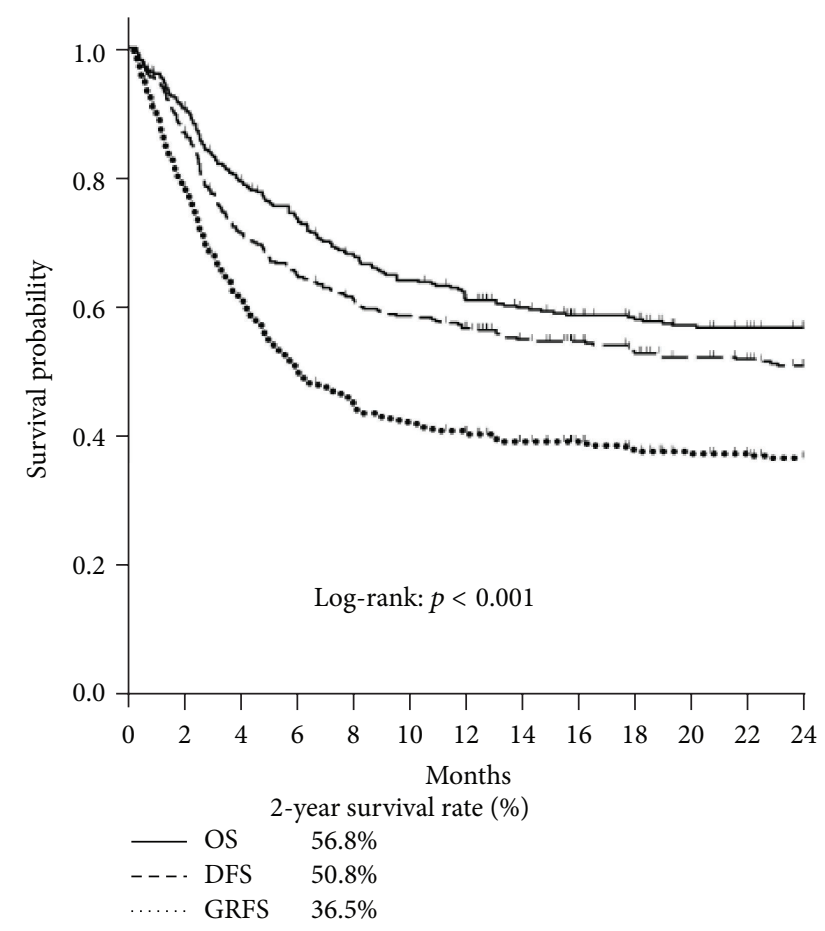

FIGURE 2: Kaplan-Meier estimates of 2-year GRFS.

GRFS (40.8\% versus 31\%) and 2-year GRFS of our patients was even similar to 1-year GRFS reported by Holtan et al. (36.5\% versus 31\%) (Figure 2) [16]. This discrepancy may be ascribed to less chronic GVHD in our patients. Generally speaking, only 30 to $40 \%$ patients receiving allogeneic HSCT

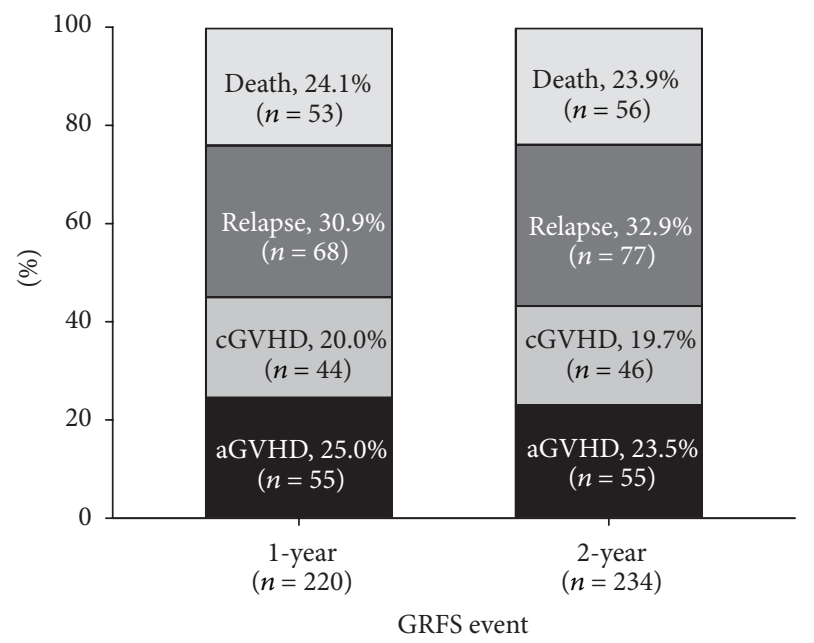

FIGURE 3: Distribution of individual components between 1-year GRFS and 2-year GRFS.

could survive to 2 years without major complications or death. Since chronic GVHD could cause significant morbidity, it is obvious that OS and DFS could not fully represent optimal recovery after allogeneic HSCT. The use of a timeto-event analysis for the survival only evaluates the time to first event without reflecting specified composite endpoints of GRFS events.

To the best of our knowledge, only one article described prognostic factors for GRFS in adult patients undergoing allogeneic HSCT [16]. In that study, high-risk disease, initial diagnosis, and year of HSCT between 2000 and 2007 were associated with poor GRFS at 1 year. In our study, age $>35$ years at HSCT, EBMT risk score > 2, hematologic malignancy at diagnosis, being male, fludarabine-based conditioning regimen, and posttransplant CMV reactivation revealed significant association with inferior 1-year and 2year GRFS in univariate regression analysis. However, in multiple regression analysis, EBMT risk score $>2$, hematologic malignancy for HSCT, and multiple transplants significantly demonstrated worse 1-year and 2-year GRFS. Additionally, male patients were significantly associated with inferior 2 year GRFS and a trend of inferior GRFS at 1 year. Our study showed results consistent with previous reports by Holtan et al. [16] regarding the prognostic factors of recipient age, advanced disease, and disease risk. However, we found that EBMT risk score provided a reliable practical tool not only to assess the pretransplant risks but also to predict the outcome of GRFS.

Pretransplant risk classification is important for predicting the outcome of patients undergoing allogeneic HSCT. The EBMT risk score was originally applied to 3,142 patients with chronic myeloid leukemia transplanted between 1989 and 1997 in Europe and then was later extended to various diagnoses of hematological disorders undergoing HSCT, including acute myeloid leukemia, acute lymphoblastic leukemia, myelodysplastic syndrome, myeloproliferative disorder, nonHodgkin lymphoma, multiple myeloma, and aplastic anemia, 
which had been repeatedly validated in $>50000$ allogeneic stem cell transplants over ten years [13, 20-23]. The risk score was determined by factors including patient age, disease stage, time interval from diagnosis to transplant, donor types, and donor recipient sex combination [13]. NRM and poor posttransplant OS were all correspondingly higher with increasing EBMT risk score. In some reported articles, they also had investigated the association between the EBMT risk score and OS, leukemia-free survival, and NRM post-HSCT [24-26]. Generally speaking, the EBMT risk score was a good predictor for OS and relapse incidence; however, the scoring system does not include parameters of acute and chronic GVHD $[24,27]$. It therefore could not be used to predict the influence of GVHD effect on OS. In our study, we also used EBMT risk score as a way to evaluate pretransplant risk on predicting GRFS. After adjusting for variables including age, hematologic malignancy at diagnosis, gender, conditioning regimen, and posttransplant CMV reactivation, EBMT risk score $>2$ was identified as a significant prognostic factor predicting a poor GRFS at either one year or two years (all $p<0.001$; HR: 1.897 and 1.835 for 1 and 2 years).

It has been claimed that EBMT risk score $\leq 2$ could explain more than $60 \%$ of the posttransplant 1- or 2-year OS and less than $30 \%$ TRM $[13,23]$. But the results of OS and TRM could not reflect a real survival without ongoing posttransplant complications. In our study, only less than $40 \%$ of patients could survive to 2 years without any complications or death (Figures 1-2) and were significantly and correspondingly lower with increasing EBMT risk score. For EBMT risk score, disease status and time interval between diagnosis and transplantation are associated with disease relapse risk. Risk factors of age, donor types, and sex matching correlate with development of GVHD and the detrimental effect of GVHD in the majority of cases often outweighed the potential benefit of GVL effect. Hence, the aforementioned 3 risk factors are associated with TRM and OS while the RFS is conceivably determined by GVHD-associated GVL effect and intensity of conditioning regimen. To our knowledge, no described scoring system could predict the GRFS currently. The extension of EBMT risk score to GRFS may help us not only integrate the risk stratification of adult allogeneic HSCT but also further assess ideal recovery following HSCT without comorbidity. Our observations warrant a prospective study for further confirmation.

During the period of adult allogeneic HSCT, pretransplant risk factors played an important role for additive impacts on GRFS, but they were not uniform and independent. According to the reported articles, posttransplant CMV reactivation would increase NRM and influence survival in patients receiving allogeneic HSCT [12, 28-30]. In addition, pretransplant comorbidities and quality of life were also important factors for HSCT recipients [31]. Hence, the additional influence of posttransplant CMV reactivation, history of type 2 diabetes mellitus, and smoking history were also evaluated in our study. Although posttransplant CMV reactivation revealed a significantly poor impact on 1- and 2-year GRFS in an univariate analysis, its influence became negligible after adjusting for variables in a multivariate Cox proportional hazards model. On the contrary, GRFS was predominantly poor with increasing EBMT risk scores (scores: 3-7). In conclusion, in the light of presentation of EBMT risk score and specified endpoint analyses of GRFS, EBMT risk scores might present a more objective approach on survival of adult allogeneic HSCT recipients.

Nowadays peripheral blood stem cell has become the predominant stem cell source for HSCT $[32,33]$ and was associated with inferior GRFS in the report by Holtan et al. [16]. In our patients with a great majority undergoing peripheral blood stem cell transplantation, the 1-year GRFS was a little better than data reported by Holtan et al. [16]. The major events in our patients occurring between 1st and 2nd year were relapse of the malignancies (Figure 3). Within this period, no more new events relating to the toxicities of HSCT, especially acute or chronic GVHD, were noted in our study. These results contribute to a lower NRM. The similar results of GRFS events were also found in the difference of prognostic factors between 1 and 2 years but no increased death rate was noted (Figure 3). These results may explain the reason why we had a better GRFS than the results reported by Holtan et al. However, our retrospective study had some limitations, including less patients receiving bone marrow transplantation and possible underestimation of clinical GVHD in the patients undergoing peripheral blood stem cell transplantation. Besides, more detailed data, such as complete data of HLA-C and DQ, infection type, and cause of death, were lacking and were not included into the analysis of prognostic model.

In conclusion, the GRFS offers an evaluation of real recovery following HSCT and is significantly influenced by some prognostic factors. EBMT risk score can act as a simple and reliable tool to predict GRFS, which provides more information than the traditional measurement of OS or DFS. All these prognostic factors could enhance our ability to optimally judge the risk and the probability of true recovery after allogeneic HSCT in adult patients.

\section{Competing Interests}

The authors declare no competing interests.

\section{Authors' Contributions}

Yao-Chung Liu and Sheng-Hsuan Chien contributed equally to this paper.

\section{Acknowledgments}

This work was partially supported by grants from the Taipei Veterans General Hospital (V104C-182), the Taiwan Clinical Oncology Research Foundation and Chong Hin Loon Memorial Cancer and Biotherapy Research Cancer, National YangMing University. 


\section{References}

[1] K. J. Thomson and K. S. Peggs, "Allogeneic transplantation in the UK: an aggregation of marginal gains?" British Journal of Haematology, vol. 163, no. 2, pp. 149-159, 2013.

[2] P. Vyas, F. R. Appelbaum, and C. Craddock, "Allogeneic hematopoietic cell transplantation for acute myeloid leukemia," Biology of Blood and Marrow Transplantation, vol. 21, no. 1, pp. 8-15, 2015.

[3] R. J. Soiffer, J. LeRademacher, V. Ho et al., "Impact of immune modulation with anti-T-cell antibodies on the outcome of reduced-intensity allogeneic hematopoietic stem cell transplantation for hematologic malignancies," Blood, vol. 117, no. 25, pp. 6963-6970, 2011.

[4] E. P. Alyea, H. T. Kim, V. Ho et al., "Comparative outcome of nonmyeloablative and myeloablative allogeneic hematopoietic cell transplantation for patients older than 50 years of age," Blood, vol. 105, no. 4, pp. 1810-1814, 2005.

[5] M. de Lima, A. Anagnostopoulos, M. Munsell et al., "Nonablative versus reduced-intensity conditioning regimens in the treatment of acute myeloid leukemia and high-risk myelodysplastic syndrome: dose is relevant for long-term disease control after allogeneic hematopoietic stem cell transplantation," Blood, vol. 104, no. 3, pp. 865-872, 2004.

[6] C. N. Bredeson, M.-J. Zhang, M.-A. Agovi et al., "Outcomes following HSCT using fludarabine, busulfan, and thymoglobulin: a matched comparison to allogeneic transplants conditioned with busulfan and cyclophosphamide," Biology of Blood and Marrow Transplantation, vol. 14, no. 9, pp. 993-1003, 2008.

[7] J.-H. Lee, Y.-D. Joo, H. Kim et al., "Randomized trial of myeloablative conditioning regimens: busulfan plus cyclophosphamide versus busulfan plus fludarabine," Journal of Clinical Oncology, vol. 31, no. 6, pp. 701-709, 2013.

[8] Y.-C. Liu, J.-P. Gau, Y.-C. Hong et al., "Large pericardial effusion as a life-threatening complication after hematopoietic stem cell transplantation-association with chronic GVHD in late-onset adult patients," Annals of Hematology, vol. 91, no. 12, pp. 19531958, 2012.

[9] Y.-C. Liu, S.-H. Chien, N.-W. Fan et al., "Risk factors for pericardial effusion in adult patients receiving allogeneic haematopoietic stem cell transplantation," British Journal of Haematology, vol. 169, no. 5, pp. 737-745, 2015.

[10] Y.-C. Liu, S.-H. Chien, N.-W. Fan et al., "Incidence and risk factors of probable and proven invasive fungal infection in adult patients receiving allogeneic hematopoietic stem cell transplantation," Journal of Microbiology, Immunology and Infection, 2015.

[11] A. H. Groll, E. Castagnola, S. Cesaro et al., "Fourth European Conference on Infections in Leukaemia (ECIL-4): guidelines for diagnosis, prevention, and treatment of invasive fungal diseases in paediatric patients with cancer or allogeneic haemopoietic stem-cell transplantation," The Lancet Oncology, vol. 15, no. 8, pp. e327-e340, 2014.

[12] P. Ljungman, R. Brand, J. Hoek et al., "Donor cytomegalovirus status influences the outcome of allogeneic stem cell transplant: a study by the European group for blood and marrow transplantation," Clinical Infectious Diseases, vol. 59, no. 4, pp. 473-481, 2014.

[13] A. Gratwohl, "The EBMT risk score," Bone Marrow Transplantation, vol. 47, no. 6, pp. 749-756, 2012.

[14] A. J. Sankoh, H. Li, and S. D’Agostino, "Use of composite endpoints in clinical trials," Statistics in Medicine, vol. 33, no. 27, pp. 4709-4714, 2014.
[15] J. Bolanos-Meade, J. Koreth, and R. Reshef, "A multicenter phase II trial randomizing novel approaches for graft-versushost disease prevention compared to contemporary controls (BMT CTN 1203)," 2015, https://clinicaltrials.gov/ct2/show/ NCT02208037.

[16] S. G. Holtan, T. E. DeFor, A. Lazaryan et al., "Composite end point of graft-versus-host disease-free, relapse-free survival after allogeneic hematopoietic cell transplantation," Blood, vol. 125, no. 8, pp. 1333-1338, 2015.

[17] M.-H. Chen, P.-C. Chen, S.-F. Jeng et al., "High perinatal seroprevalence of cytomegalovirus in northern Taiwan," Journal of Paediatrics and Child Health, vol. 44, no. 4, pp. 166-169, 2008.

[18] E. D. Thomas, R. Storb, R. A. Clift et al., "Bone-marrow transplantation (second of two parts)," New England Journal of Medicine, vol. 292, no. 17, pp. 895-902, 1975.

[19] A. H. Filipovich, D. Weisdorf, S. Pavletic et al., "National Institutes of Health Consensus Development Project on criteria for clinical trials in chronic graft-versus-host disease. I. Diagnosis and staging working group report," Biology of Blood and Marrow Transplantation, vol. 11, no. 12, pp. 945-956, 2005.

[20] A. Gratwohl, J. Hermans, J. M. Goldman et al., "Risk assessment for patients with chronic myeloid leukaemia before allogeneic blood or marrow transplantation," The Lancet, vol. 352, no. 9134, pp. 1087-1092, 1998.

[21] J. O. Armitage, "Bone marrow transplantation," The New England Journal of Medicine, vol. 330, no. 12, pp. 827-838, 1994.

[22] J. Sierra, B. Storer, J. A. Hansen et al., "Transplantation of marrow cells from unrelated donors for treatment of high-risk acute leukemia: the effect of leukemic burden, donor HLAmatching, and marrow cell dose," Blood, vol. 89, no. 11, pp. 42264235, 1997.

[23] A. Gratwohl, M. Stern, R. Brand et al., "Risk score for outcome after allogeneic hematopoietic stem cell transplantation: a retrospective analysis," Cancer, vol. 115, no. 20, pp. 4715-4726, 2009.

[24] K. Rezvani, E. J. Kanfer, D. Marin et al., "EBMT risk score predicts outcome of allogeneic hematopoietic stem cell transplantation in patients who have failed a previous transplantation procedure," Biology of Blood and Marrow Transplantation, vol. 18, no. 2, pp. 235-240, 2012.

[25] T. H. Terwey, P. G. Hemmati, P. Martus et al., "A modified EBMT risk score and the hematopoietic cell transplantation-specific comorbidity index for pre-transplant risk assessment in adult acute lymphoblastic leukemia," Haematologica, vol. 95, no. 5, pp. 810-818, 2010.

[26] T. Lodewyck, M. Oudshoorn, B. van der Holt et al., "Predictive impact of allele-matching and EBMT risk score for outcome after T-cell depleted unrelated donor transplantation in poorrisk acute leukemia and myelodysplasia," Leukemia, vol. 25, no. 10, pp. 1548-1554, 2011.

[27] A. Jalali, K. Alimoghaddam, M. Mahmoudi et al., "The EBMT risk score in the presence of graft versus host disease in allogeneic stem cell transplantation in adult acute myelogenous leukemia: a multistate model for competing risks," International Journal of Hematology-Oncology and Stem Cell Research, vol. 8, no. 3, pp. 1-11, 2014.

[28] J. Liu, J. Kong, Y. J. Chang et al., "Patients with refractory cytomegalovirus (CMV) infection following allogeneic haematopoietic stem cell transplantation are at high risk for CMV disease and non-relapse mortality," Clinical Microbiology and Infection, vol. 21, no. 12, pp. 1121.e9-1121.e15, 2015. 
[29] P. Ljungman, M. Hakki, and M. Boeckh, "Cytomegalovirus in hematopoietic stem cell transplant recipients," Infectious Disease Clinics of North America, vol. 24, no. 2, pp. 319-337, 2010.

[30] P. Ljungman, "The role of cytomegalovirus serostatus on outcome of hematopoietic stem cell transplantation," Current Opinion in Hematology, vol. 21, no. 6, pp. 466-469, 2014.

[31] B. K. Hamilton, A. D. Law, L. Rybicki et al., "Prognostic significance of pre-transplant quality of life in allogeneic hematopoietic cell transplantation recipients," Bone Marrow Transplantation, vol. 50, no. 9, pp. 1235-1240, 2015.

[32] M. C. Pasquini, Z. Wang, and M. M. Horowitz, "2010 report from the Center for International Blood and Marrow Transplant Research (CIBMTR): current uses and outcomes of hematopoietic cell transplants for blood and bone marrow disorders," Clinical Transplants, pp. 87-105, 2010.

[33] U. Holtick, M. Albrecht, J. M. Chemnitz et al., "Comparison of bone marrow versus peripheral blood allogeneic hematopoietic stem cell transplantation for hematological malignancies in adults-a systematic review and meta-analysis," Critical Reviews in Oncology/Hematology, vol. 94, no. 2, pp. 179-188, 2015. 

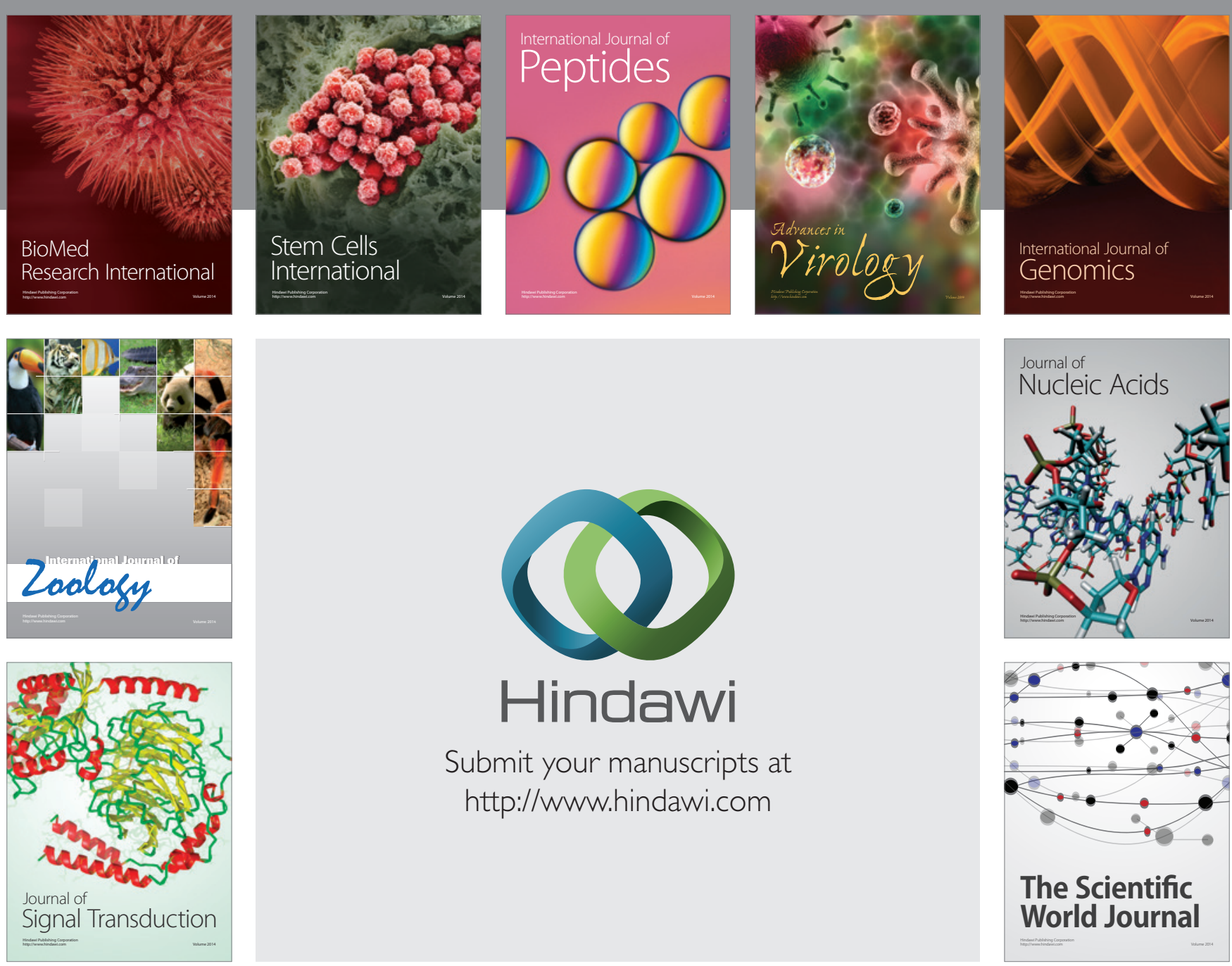

Submit your manuscripts at

http://www.hindawi.com
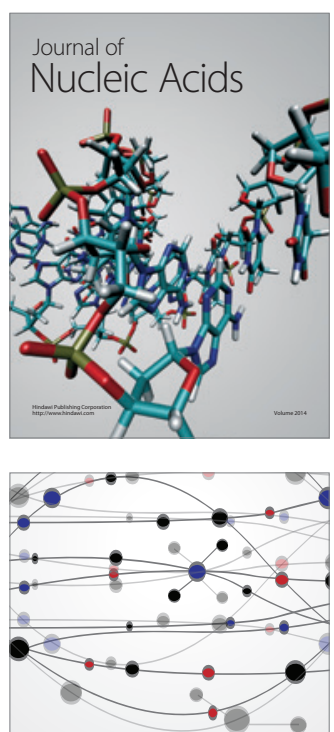

The Scientific World Journal
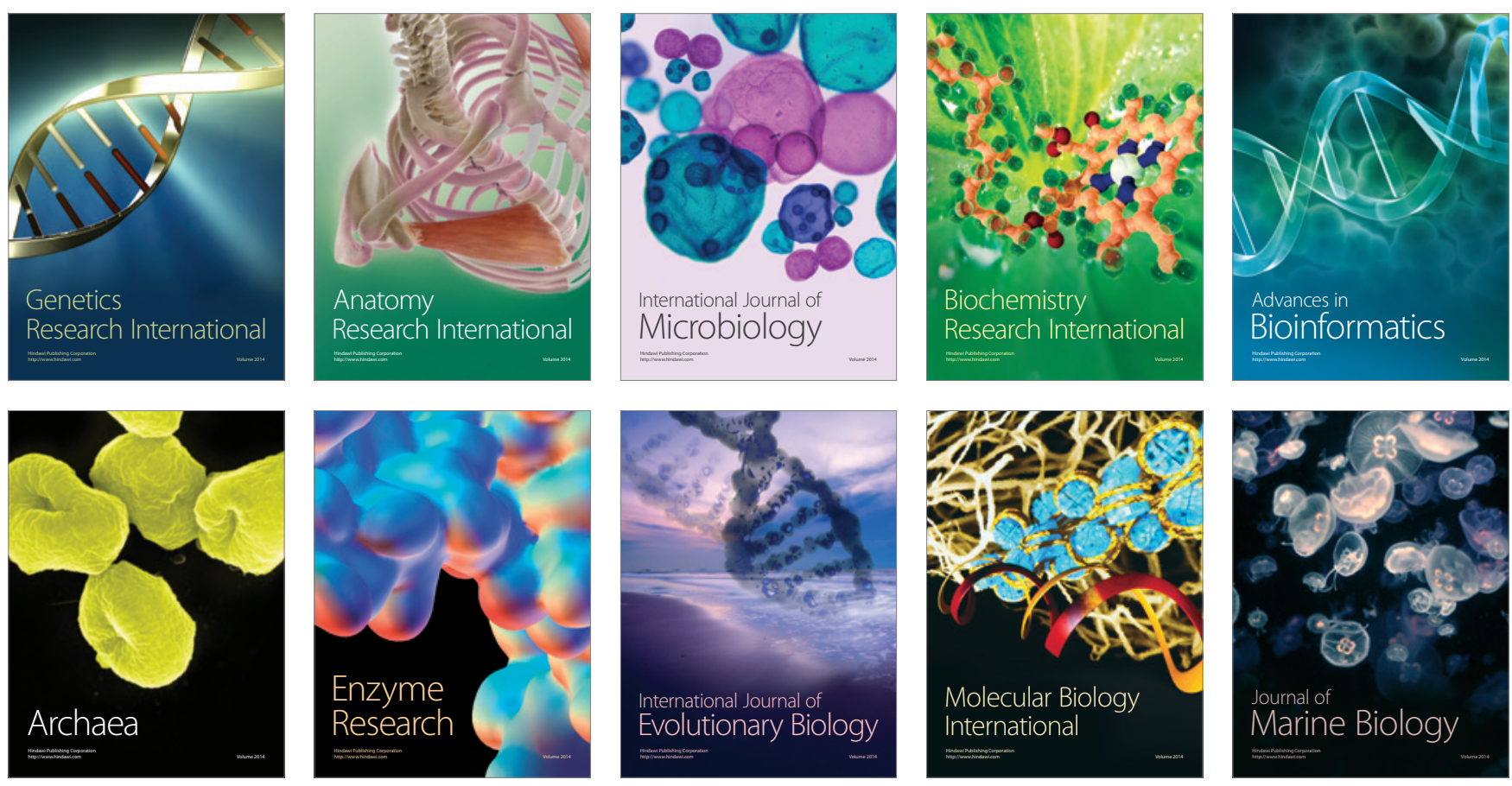